\title{
Immediate laser-induced hemostasis in anticoagulated rats subjected to oral soft tissue surgery: a double-blind study
}

\begin{abstract}
Flavio Halak de Oliveira CAMPOS(a) Lorraine Braga FERREIRA ${ }^{(b)}$ Marcelo Munhoes ROMANO(c) Maria Stella MOREIRA(a) Carlos de Paula EDUARDO(d) Karen Müller RAMALHO(a)
\end{abstract} (a) Universidade lbirapuera - UNIB, School of
Dentistry, São Paulo, SP, Brazil.

(b) Universidade de São Paulo - USP, School of Dentistry, Department of Biomaterials, São Paulo, SP, Brazil.

(c) Universidade de São Paulo - USP, School of Dentistry, Department of Stomatology, São Paulo, SP, Brazil.

(d) Universidade de São Paulo - USP, School of Dentistry, Department of Restorative

Declaration of Interests: The authors certify that they have no commercial or associative interest that represents a conflict of interest in connection with the manuscript.

Corresponding Author:

Karen Muller Ramalho

E-mail: karenramalho@hotmail.com

Submitted: July 31, 2017

Accepted for publication: April 13, 2018

Last revision: May 07, 2018

https://doi.org/10.1590/1807-3107bor-2018.vol32.0056 Dentistry, São Paulo, SP, Brazil.

Abstract: Given the growing trend towards medical indications for continuous use of anticoagulants, the number of patients on these medications continues to rise. The management of patients on oral anticoagulants requiring oral surgical procedures has aroused much controversy. Changes in an anticoagulation regimen are associated with an increased risk of thromboembolism. However, it seems logical and advantageous for the patients' health if surgery could be performed without any change to the anticoagulation therapy. In dentistry, high-power lasers have been poorly explored in this field. The hemostatic properties of high-power lasers could be helpful during oral soft tissue surgeries in anticoagulated patients. The aim of this study was to compare bleeding time in anticoagulated rats after lingual frenectomy performed with a scalpel or diode laser with bleeding time in healthy animals. Twenty-four male Wistar rats were assigned to four groups ( $n=6$ ): (CS) Control-Scalpel Surgery; (AS) Anticoagulated-Scalpel Surgery; (CL) Control-Laser (diode laser $810 \mathrm{~nm} / 1.5 \mathrm{~W}$ ) Surgery; and (AL) Anticoagulated-Laser Surgery (diode laser $810 \mathrm{~nm} / 1.5 \mathrm{~W}$ ). Warfarin administration was used to induce anticoagulation. Blood was blotted every 30 seconds with filter paper until bleeding stopped to verify bleeding time. Two blinded researchers performed the surgeries and collected the bleeding time data. Diode laser surgery led to complete hemostasis in rats during and after lingual frenectomy. Zero bleeding was assessed during surgeries and after diode laser surgeries in anticoagulated rats. Laser-induced hemostasis offered an alternative solution to the controversial issue of intraoperative and postoperative bleeding control in patients on anticoagulation therapy.

Keywords: Laser Coagulation; Warfarin; Surgery, Oral.

\section{Introduction}

The need to deal with anticoagulated patients during dental care has become increasingly frequent. At present, indications for antithrombotic therapy include coronary artery disease, vascular thromboembolism, myocardial infarction, cerebrovascular ischemic attacks, valvular disorders, prosthetic heart valves, atrial fibrillation, deep vein thrombosis, and pulmonary embolism. ${ }^{1}$ The most commonly used anticoagulants are heparins, antiplatelet drugs, and thrombolytic agents; ${ }^{2}$ and hemorrhage 
is the major adverse effect of oral anticoagulants. ${ }^{2}$ In the past, the approach used to be to discontinue or to change the anticoagulation regimen before performing an oral surgery. In 1957, Ziffer et al. ${ }^{3}$ reported serious postoperative bleeding after oral surgery in anticoagulated patients. Since then, the customary practice has been to discontinue or reduce anticoagulation therapy. The management of patients on oral anticoagulants requiring oral surgical procedures has been riven by controversy. ${ }^{4}$ Although some patients have stopped taking antithrombotic medications before dental surgery without developing serious side effects, ${ }^{5}$ others have suffered complications, such as thrombosis and embolism., ${ }^{4,6}$ Several incidents have been reported in the literature, including deaths after anticoagulation therapy discontinuation. ${ }^{6,7,8,9}$

Oral anticoagulation therapy status was initially monitored by the one-stage prothrombin time (PT) test. In 1985, the INR (international normalized ratio) was introduced to standardize PT reports by converting the PTratiointo INRaccording to the formula: $I N R=(f \triangle R$ Ratio) ISI. Hence, the intensity of anticoagulation therapy is safely and accurately measured by this calibration method. ${ }^{10,11,12}$ For most therapeutic indications, i.e., atrial fibrillation, myocardial infarction, and deep venous thromboembolism, the targeted goal is to obtain an INR with a therapeutic range between 2 and $3 .^{12}$ The American Heart Association/American College of Cardiology and the European Society of Cardiology/British Committee for Standards in Hematology recommend an INR range from 2.5 to 3.5/4 for mechanical heart valves. ${ }^{13}$

Changes in the anticoagulation regimen are associated with an increased risk of thromboembolism. ${ }^{14,15}$ Many studies have demonstrated that oral surgical procedures can be safely performed if the INR is within the therapeutic range (INR $<4$ ), and local hemostatic agents such as surgical hemostasis and primary closure with sutures are applied afterwards to prevent postoperative bleeding. ${ }^{2}$

With the introduction of lasers into the field of dentistry, a new treatment modality has been made available to oral surgeons. The inherent advantages of lasers are hemostasis, decreased charring, and lower postoperative pain. ${ }^{16,17}$ The hemostatic nature of lasers, based on the photothermal or photochemical interaction of light and matter, is of great value in oral surgery. It allows surgery to be performed more precisely and accurately because the surgeon has better visibility of the surgical site. Consequently, operative time is reduced and postoperative hemorrhage is minimized. ${ }^{2}$ Laser application could be the key to diminishing intraoperative and postoperative hemorrhage and decreasing postoperative symptoms, offering an alternative for the management of oral surgery in anticoagulated patients. High-power lasers with different wavelengths have been applied successfully in surgeries of anticoagulated patients in different medical specialties, without the need to interrupt anticoagulation therapy. ${ }^{18,19}$ In Dentistry, however, there are few reports on the use of highpower lasers. The aim of this study was to compare bleeding time in anticoagulated rats after lingual frenectomy performed with a scalpel or high-power diode laser with bleeding time in healthy animals.

The null hypothesis is that high-power laser does not interfere in bleeding time after lingual frenectomy in anticoagulated rats.

\section{Methodology}

The experimental protocol for this study was approved by the Ethics Committee on Animal Research of the University of São Paulo, Brazil (process \#19/2014). The principles of laboratory animal care (NIH publication 85-23, 1985) and national laws on animal use were complied with in the present study. The animals were kept in individual and ventilated plastic cages at $22^{\circ} \mathrm{C}$ on a 12-h light/dark cycle and received standard rodent chow and water ad libitum during the experimental phase of the study. The animals were kept in individual plastic cages for 72 hours, during which time warfarin was diluted in the drinking water. Each animal was monitored closely for water intake throughout that period.

Twenty-four 12-week-old male Wistar rats weighing 280-300 g each were used in the experiments. The animals were split into four groups $(n=6)$ : Control-Scalpel Surgery (CS); AnticoagulatedScalpel Surgery (AS); Control-Laser Surgery (CL); and Anticoagulated-Laser Surgery (AL). 


\section{Warfarin administration}

In anticoagulated animals, warfarin was administered according to Illanes et al. ${ }^{20}$ Warfarin (Coumadin ${ }^{\circledR}$, Bristol-Myers-Squibb) $(2.5 \mathrm{mg}$ ) was dissolved in $800 \mathrm{~mL}$ of drinking water, and 72 hours before surgery the animals were given water containing warfarin. Each animal was provided with $400 \mathrm{~mL}$ of drinking water for 72 hours. The volume of water drunk by the animal was also evaluated, with the purpose of maintaining standardization among the experimental animals. The control animals were provided with the same volume of drinking water, but without warfarin. As the animals were kept in individual cages, the volume of water consumed was recorded as follows: Initial volume in the bottle - Final volume in the bottle = Consumed volume.

\section{INR (International Normalized Ratio) validation.}

To confirm the change in INR after warfarin administration, blood samples were collected from the tail of the animals before surgery, which were evaluated by using a point-of-care coagulometer (CoaguChek XS®, Roche).

\section{Surgical procedure}

The animals were anesthetized with an intramuscular injection of $0.86 \mathrm{~mL} / \mathrm{kg}$ of ketamine and $0.53 \mathrm{~mL} / \mathrm{kg}$ of xylazine. The base of the labial frenum of each animal in the CS and AS groups was cut by using a disposable scalpel (\#15, Embramed, São Paulo, SP - Brazil) (Figure 1). However, in the CL and AL groups, the base of the labial frenum was cut by using an 810-nm high-power diode laser (Thera Laser Surgery®, DMC São Carlos, Brazil). A 1.5-W power was used in the surgery, which was performed by means of an optical fiber with a $600-\mu \mathrm{m}$ core diameter. A researcher who was blinded to the group to which the animal had been allocated performed all the surgeries.

\section{Bleeding time evaluation}

After frenectomy, bleeding time was evaluated by another blinded researcher according to Konishi et al.

${ }^{21}$ Blood was blotted with filter paper every 30 seconds until the bleeding stopped (Figure 1). The animals were followed up/monitored for 15 minutes to evaluate possible late bleeding.

\section{Statistical analyses}

The nonparametric Kruskal-Wallis test was used to compare the data. The Student-Newman-Keuls method was used as post-hoc test to compare the median of the groups. BioStat 5.0.1 was used for the statistical analyses. The significance level was set at $5 \%$.

\section{Results}

\section{Anticoagulation induction protocol}

Warfarin administration ${ }^{18}$ proved to be effective in inducing anticoagulation in rats in the present study, as shown by INR values obtained after warfarin therapy (Table 1 ). INR values were $<1.1$ in the control groups and $>5$ in anticoagulated animals. As the animals were kept in individual cages, the volume of water intake could be verified. The mean volume $( \pm \mathrm{SD})$ of water consumed in all cages in 3 days (72 h) was $302 \pm 14 \mathrm{~mL}$. There was a negligible variation in water intake among animals in this study.

\section{Bleeding time analysis}

Bleeding time analysis showed a significant difference $\left(\chi^{2}=7.815, \mathrm{p} \leq 0.05\right)$ between scalpel surgery and laser surgery (Table 2 ) in anticoagulated and control animals. After scalpel surgery, anticoagulated animals showed a median bleeding time of 637.5 seconds, compared to 237.6 seconds in control animals. Diode laser surgery led to complete hemostasis in rats during and after lingual frenectomy. No bleeding was observed during and after surgeries in control and anticoagulated rats after diode laser surgeries (Table 2, Figure 1). Laser proved to be an effective tool for controlling bleeding, particularly in anticoagulated animals, among which bleeding time was higher (Table 2). There was no late bleeding in any animals within 15 minutes after the end of surgery. 


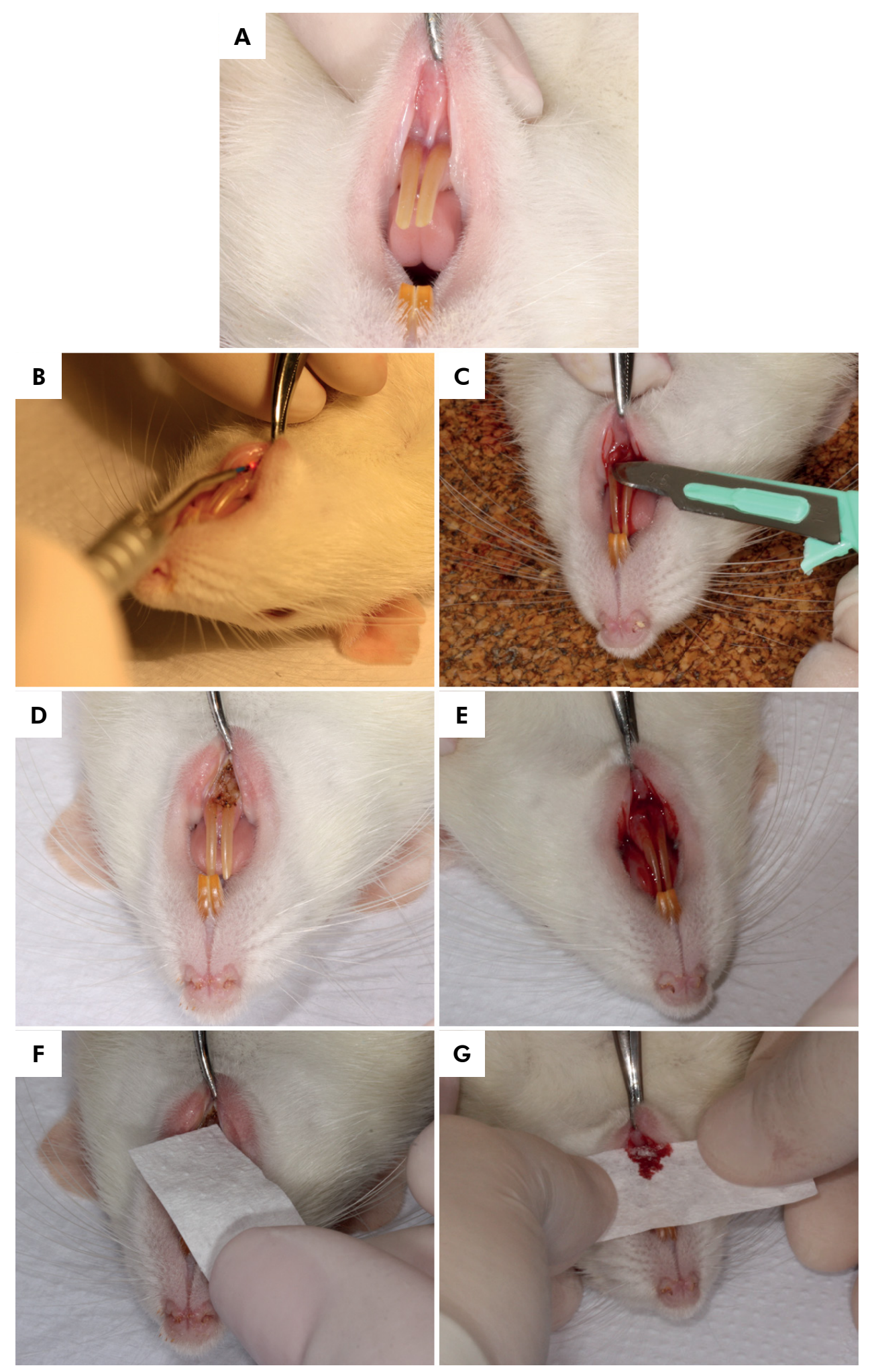

Figure 1. A - Rat labial frenum before surgery; B - Labial frenectomy being performed by high-power diode laser; C- Labial frenectomy being performed by scalpel; D - Rat labial frenum immediately after frenectomy performed by diode high-power laser; E - Rat labial frenum immediately after frenectomy performed by scalpel; $F$ - Bleeding time evaluation with filter paper blotted every 30 seconds, above surgical site, in the high-power diode laser group; $G$ - Bleeding time evaluation with filter paper blotted every 30 seconds, above surgical site, in the Scalpel group. 
Table 1. Mean and standard deviation of INR values in control and experimental groups.

\begin{tabular}{lccc}
\hline Control-scalpel surgery (CS) & Control-laser surgery (CL) & Anticoagulated-scalpel surgery (AS) & Anticoagulated-laser surgery (AL) \\
\hline $1.1 \pm 0.006$ & $1.0 \pm 0.008$ & $5.0 \pm 1.1$ & $5.5 \pm 1.1$ \\
\hline
\end{tabular}

Table 2. Mean and standard deviation of bleeding time (seconds).

\begin{tabular}{|c|c|c|c|c|c|}
\hline Groups & Mean (+ SD) & Median & First Quartile & Third Quartile & $I Q R$ \\
\hline Scalpel surgery - control animals & $231.7 \pm 85$ & $237.5^{a}$ & 168.7 & 298.7 & 130 \\
\hline Scalpel surgery - anticoagulated animals & $614, .2 \pm 314.3$ & $637.5^{a}$ & 333.7 & 900 & 566.2 \\
\hline Laser surgery - control animals & 0 & $0^{\mathrm{b}}$ & 0 & 0 & 0 \\
\hline Laser surgery - anticoagulated animals & 0 & $0^{b}$ & 0 & 0 & 0 \\
\hline
\end{tabular}

The same letters indicate absence of statistical differences $(p \geq 0.05)$.

\section{Discussion}

The present study showed significant results that could support the use of high-power lasers in soft tissue surgeries on anticoagulated patients. The null hypothesis was rejected, since high-power laser could interfere in bleeding time after lingual frenectomy in anticoagulated rats. In our double-blind study, diode laser induced immediate hemostasis during frenectomy in anticoagulated rats. With the protocol adopted to induce anticoagulation in Wistar rats ${ }^{20}$ the INR was in the range of 5.0-5.5. Thus, exacerbated bleeding was observed during the surgeries of these animals when compared to the control group.

To make sure that all animals would drink a very similar volume of water containing warfarin, all of them were kept in individual cages during the experimental period (72 hours). In fact, the rats should not be housed individually, once isolation can alter many behavioral and biochemical parameters, being extremely stressful for animals. However, as mentioned previously, isolation of the animals only during the experimental phase of the study (72 hours) was mandatory for the control of water intake by each animal. In this study, some of the effects of individual housing were mitigated by ensuring visual (e.g., use of transparent walls), auditory, and olfactory contact with other rats during the experimental phase. ${ }^{22}$

In a dental clinic, exacerbated bleeding - especially during dental procedures performed by a general dentist - may cause some panic and fear. Furthermore, there are important arguments advocating that anticoagulation therapy should not be discontinued when patients have to be subjected to oral soft tissue surgery.

As evidenced in the literature, there are often some barriers to the dental treatment of patients with inherent bleeding disorders or patients on anticoagulation therapy. ${ }^{23}$ These barriers could originate from patients or clinicians. Patient-related barriers include fear of bleeding during dental procedures or during tooth brushing and lack of confidence in the dentist, ${ }^{24,25}$ while dentists (especially general dentists) may often feel inadequately trained to handle these patients in case of bleeding complications. Consequently, dental care of these patients is frequently neglected, resulting in a high level of untreated dental disease. ${ }^{23} \mathrm{~A}$ retrospective study by Rasaratnam et al. ${ }^{23}$ showed that patients with bleeding disorders had a high level of dental treatment needs, with a high prevalence of decay and gum disease, which accounted for $83 \%$ and $46.7 \%$ of their cohort and which is largely related to the patients' irregular dental attendance pattern. Patients' apprehension and dentists' fear are important factors to be considered. ${ }^{23}$

The management of patients on oral anticoagulation therapy who need oral surgical treatment has been surrounded by controversy. ${ }^{4}$ To date, there has been a lack of consensus on how to manage the anticoagulation regimen of patients on coumarins who are faced with the need to undergo oral surgery. ${ }^{4}$ Options include maintaining the anticoagulation therapy, reducing the dose of oral anticoagulants, and discontinuing oral anticoagulation therapy altogether. 
Complete cessation of warfarin administration in patients requiring anticoagulation can lead to severe thromboembolism, causing severe neurological events or even death. ${ }^{4,6,7,8,26}$ Interruption of therapy may expose patients to a greater risk resulting from the condition for which they are being treated prophylactically. ${ }^{27}$ Furthermore, discontinuation may create a transient hypercoagulable state, thus exposing patients to a higher risk of thromboembolic events, ${ }^{14,28}$ an issue that has raised a lot of controversy, though. ${ }^{29}$ The possibility of severe postoperative bleeding is a concern of any patient taking anticoagulants. However, discontinuation of anticoagulation therapy for a certain period before surgery has the potential to place the patient at greater risk of life-threatening thromboembolism. ${ }^{26}$ Furthermore, there is evidence that thrombosis may actually be more likely to take place because of the hypercoagulable state that may occur after cessation of oral anticoagulation therapy, ${ }^{14,28,30}$ although some authors have proposed discontinuing warfarin for $4-5$ days in cases of patients at low risk of thromboembolism. . $931,32,33$

However, it seems logical and advantageous for the patients' health if oral surgeries could be performed without any change in the anticoagulation regimen. Despite the potential risk of bleeding complications, most patients with inherent bleeding disorders, or those who take anticoagulants, can be properly managed at a general practice. ${ }^{20} \mathrm{~A}$ close relationship between the hematology team and the dentist is essential to minimize the risk of complications in these patients. ${ }^{34}$

Notwithstanding the benefits of high-power lasers, such as hemostasis, decreased charring, better visualization of the surgical site, and lower postoperative pain ${ }^{16,17}$ they have been poorly explored in dentistry, especially regarding their application to patients with bleeding disorders or on anticoagulation therapy who need to undergo oral surgeries. Little information could be found in the literature and there was no study proving the benefits of highpower lasers to patients at risk of preoperative and postoperative bleeding during minor oral surgeries.

In 1990, Chellappah and Loh ${ }^{35}$ published a case report describing a hemophilic patient with acute pericoronitis. The operculum associated with an erupting molar tooth was excised using a $\mathrm{CO}_{2}$ laser. Preoperative or postoperative infusion of Factor VIII was not required. According to the authors, laser offered an alternative method for providing minor surgical care to hemophilic patients. ${ }^{35}$

In the study of Rasaratnam et al., ${ }^{23}$ in which they discussed the risk-based management of dental procedures in patients with inherent bleeding disorder, they pointed out that the literature has not taken into account the evolving developments in dental practice, such as the use of lasers, which might contribute to reducing the risk of bleeding. ${ }^{23,35}$ That could have occurred because of the lack of information in the literature about high-power laser in this field. The present double-blind study showed that diode laser could induce immediate hemostasis in anticoagulated rats subjected to oral soft tissue surgery. Both control and anticoagulated animals had zero bleeding when surgeries were performed using diode laser.

The minor oral surgery model adopted in the present study consisted of labial frenectomy, a surgical procedure for excising a frenum or frenulum, such as the excision of the lingual frenum from its attachment into the mucoperiosteal covering of the alveolar process to correct ankyloglossia. The surgery is a straightforward and quite common dental procedure performed in both children and adults. Therefore, other less invasive surgeries that involve oral soft tissues could be easily performed with laser in anticoagulated patients. Oral soft tissues have a large amount of rather small vessels and capillaries, which are traumatized during surgery. ${ }^{2}$ Depending on beam types and power density, laser can seal blood vessels up to a $0.5-\mathrm{mm}$ diameter. ${ }^{34}$ The use of laser-assisted oral soft tissue surgeries can achieve hemostasis and prompt blood clot formation. ${ }^{2}$

General dentists may feel safer and more comfortable using high-power lasers for minor oral soft tissue surgeries. Another great advantage of using laser is its greater acceptance by patients. ${ }^{2}$ Some procedures performed by general dentists could be classified as posing moderate bleeding risk to anticoagulated patients. According to Rasaratnam et al, ${ }^{23}$ gum trauma or preparations beyond the gum margin could be associated with a risk of trauma and subsequent bleeding complications, which are classified as 
moderate bleeding risk. Thus, the use of high-power lasers could be extremely beneficial to these patients. This study showed that laser-induced hemostasis could improve current surgical options for the treatment of patients on anticoagulation therapy without changing the medication regimen. Laserassisted oral surgery can be performed irrespective of the variation in INR values due to the excellent hemostasis achieved with surgical laser systems. Thus, there is no need to discontinue or reduce anticoagulation medication, exposing the patient to the risk of thromboembolic complications. ${ }^{2}$ In view of the growing trend towards medical indications for continuous use of anticoagulants, the number of patients on these medications will continue to rise. Laser-induced hemostasis offers an alternative

\section{References}

1. Tierney LM, Mcphee SJ, Paradakis MA. Current medical diagnosis \& treatment. 44th ed. New York: Lenge; 2005.

2. Chrysikopoulos S, Papaspyridakos P, Eleftheriades E. Laser-assisted oral and maxillofacial surgery for patients on anticoagulant therapy in daily practice. J Oral Laser Appl. 2006;6(2):79-88.

3. Ziffer AM, Scopp IW, Beck J, Baum J, Berger AR. Profound bleeding after dental extractions during dicumarol therapy. N Engl J Med. 1957 Feb;256(8):351-3. https://doi.org/10.1056/NEJM195702212560806

4. Eichhorn W, Burkert J, Vorwig O, Blessmann M, Cachovan G, Zeuch J et al. Bleeding incidence after oral surgery with continued oral anticoagulation. Clin Oral Investig. 2012 Oct;16(5):1371-6. https://doi.org/10.1007/s00784-011-0649-1

5. Mulligan R, Weitzel KG. Pretreatment management of the patient receiving anticoagulant drugs. J Am Dent Assoc. 1988 Sep;117(3):479-83. https://doi.org/10.1016/S0002-8177(88)73021-4

6. Marshall J. Rebound phenomena after anticoagulant therapy in cerebrovascular disease. Circulation. 1963 Sep;28(3):329-32. https://doi.org/10.1161/01.CIR.28.3.329

7. Akbarian M, Austen G, Yurchak PM, Scannell JG. Thromboembolic complications of prosthetic cardiac valves. Circulation. 1968 May;37(5):826-31. https://doi.org/10.1161/01.CIR.37.5.826 solution to the controversial issue of intraoperative and postoperative bleeding control in patients on anticoagulation therapy. ${ }^{2}$

\section{Conclusion}

In the present study, diode laser $(810 \mathrm{~nm})$ at $1.5 \mathrm{~W}$ induced immediate hemostasis in anticoagulated rats undergoing oral soft tissue surgery (labial frenectomy).

\section{Acknowledgments}

The authors would like to thank $\mathrm{CNPq}$ (\#305574/2008-6) for the financial support, professor Victor Elias Arana Chavez for making the Oral Biology Laboratory (FOUSP) available for this research, and professor Flávia Gonçalves for the statistical support.
8. Behrman SJ, Wright IS. Dental surgery during continuous anticoagulant therapy. JAMA. 1961 Feb;175(6):483-8. https://doi.org/10.1001/jama.1961.03040060057012

9. Cosgriff SW. Chronic anticoagulant therapy in recurrent embolism of cardiac origin. Ann Intern Med. 1953 Feb;38(2):278-87. https://doi.org/10.7326/0003-4819-38-2-278

10. Loeliger EA, Besselaar AM, Lewis SM. Reliability and clinical impact of the normalization of the prothrombin times in oral anticoagulant control. Thromb Haemost. 1985 Feb;53(1):148-54.

11. Terezhalmy GT, Lichtin AE. Antithrombotic, anticoagulant, and thrombolytic agents. Dent Clin North Am. 1996 Jul;40(3):649-64.

12. Hirsh J, Poller L. The international normalized ratio: a guide to understanding and correcting its problems. Arch Intern Med. 1994 Feb;154(3):282-8. https://doi.org/10.1001/archinte.1994.00420030084008

13. Hirsh J, Fuster V, Ansell J, Halperin JL. American Heart Association/American College of Cardiology Foundation guide to warfarin therapy. Circulation. 2003 Apr;107(12):1692-711. https://doi.org/10.1161/01.CIR.0000063575.17904.4E

14. Poller L, Thomson J. Evidence for

"rebound" hypercoagulability after stopping anticoagulants. Lancet. 1964 Jul;2(7350):62-4. https://doi.org/10.1016/S0140-6736(64)90067-4

15. Jeske AH, Suchko GD. Lack of a scientific basis for routine discontinuation of oral anticoagulation therapy before dental treatment. J Am Dent Assoc. 2003 Nov;134(11):1492-7. https://doi.org/10.14219/jada.archive.2003.0080 
16. Aoki A, Sasaki KM, Watanabe H, Ishikawa I. Lasers in nonsurgical periodontal therapy. Periodontol 2000. 2004;36(1):59-97. https://doi.org/10.1111 /j.1600-0757.2004.03679.xPMID:15330944

17. Rossmann JA, Cobb CM. Lasers in periodontal therapy. Periodontol 2000. 1995 Oct;9(1):150-64. https://doi. org/10.1111/j.1600-0757.1995.tb00062.x PMID:9567984

18. Dayani pn, Grand MG. Maintenance of warfarin anticoagulation for patients undergoing vitroretinal surgery. Trans Am Ophthalmol Soc. 2006;104(11):149-60. https://doi.org/10.1001/archopht.124.11.1558

19. Riesenman PJ, Fritas DJ, Konigsberg SG, Kasirajan K. Noninterruption of warfarin therapy is safe and does not compromise outcome in patients undergoing endovenous laser therapy (EVLT). Vasc Endovascular Surg. 201;45(6):524-6. https://doi.org/10.1177/1538574411414302

20. Illanes S, Zhou W, Heiland S, Markus Z, Veltkamp R. Kinetics of hematoma expansion in murine warfarin-associated intracerebral hemorrhage. Brain Res. 2010 Mar;1320:135-42. https://doi.org/10.1016/j.brainres.2010.01.015

21. Konishi N, Hiroe K, Kawamura M. Synergistic effect of a factor Xa inhibitor, TAK-442, and antiplatelet agents on whole blood coagulation and arterial thrombosis in rats. Thromb Res. 2010 Aug;126(2):124-9. https://doi.org/10.1016/i.thromres.2010.04.005

22. Hurst JL, Barnard CJ. Nevison, West CD. Housing and welfare in laboratory rats: welfare implications of isolation and social contact among caged males. Anim Welf. 1997;6:329-47.

23. Rasaratnam L, Chowdary P, Pollard D, Subel B, Harrington C, Darbar UR. Risk-based management of dental procedures in patients with inherited bleeding disorders: Development of a Dental Bleeding Risk Assessment and Treatment Tool (DeBRATT). Haemophilia. 2017 Mar;23(2):247-54. https://doi.org/10.1111/hae.13122

24. Dougall A, Fiske J. Access to special care dentistry, part 5. Safety. Br Dent J. 2008 Aug;205(4):177-90. https://doi.org/10.1038/sj.bdj.2008.693

25. Smith MP, Savidge G, Fiske J, McGeoch RJ. The treatment needs of adults with inherited bleeding disorders. J Disabil Oral Health. 2002 Oct;3(2):59-61.
26. Tulloch J, Wright IS. Long-term anticoagulant therapy; further experiences. Circulation. 1954 Jun;9(6):823-34. https://doi.org/10.1161/01.CIR.9.6.823

27. Kuwada SK, Balm R, Gostout CJ. The risk of withdrawing chronic anticoagulation because of acute GI bleeding. Am J Gastroenterol. 1996 Jun;91(6):1116-9.

28. Grip L, Blombäck M, Schulman S. Hypercoagulable state and thromboembolism following warfarin withdrawal in post-myocardialinfarction patients. Eur Heart J. 1991 Nov;12(11):1225-33. https://doi.org/10.1093/eurhearti/12.11.1225

29. Tardy B, Tardy-Poncet B, Laporte-Simitsidis S, Mismetti P, Decousus H, Guyotat D et al. Evolution of blood coagulation and fibrinolysis parameters after abrupt versus gradual withdrawal of acenocoumarol in patients with venous thromboembolism: a double-blind randomized study. Br J Haematol. 1997 Jan;96(1):174-8. https://doi.org/10.1046/i.1365-2141.1997.8752506.x

30. Genewein U, Haeberli A, Straub PW, Beer JH. Rebound after cessation of oral anticoagulant therapy: the biochemical evidence. Br J Haematol. 1996 Feb;92(2):479-85. https://doi.org/10.1046/i.1365-2141.1996.d01-1499.x

31. Hirsh J, Fuster V, Ansell J, Halperin JL; American Heart Association/ American College of Cardiology Foundation. American Heart Association/American College of Cardiology Foundation guide to warfarin therapy. J Am Coll Cardiol. 2003 May;41 (9):1633-52. https://doi.org/10.1016/S0735-1097(03)00416-9

32. Kearon C, Hirsh J. Management of anticoagulation before and after elective surgery. N Engl J Med. 1997 May;336(21):1506-11. https://doi.org/10.1056/NEJM199705223362107

33. Jafri SM, Mehta TP. Periprocedural management of anticoagulation in patients on extended warfarin therapy. Semin Thromb Hemost. 2004 Dec;30(6):657-64. https://doi.org/10.1055/s-2004-861508

34. Anderson JA, Brewer A, Creagh D, Hook S, Mainwaring J, McKernan A et al. Guidance on the dental management of patients with haemophilia and congenital bleeding disorders. Br Dent J. 2013 Nov;215(10):497-504. https://doi.org/10.1038/sj.bdj.2013.1097

35. Chellappah NK, Loh HS. Laser therapy for a haemophiliac: case report. Aust Dent J. 1990 Apr;35(2):121-4. https://doi.org/10.1111/j.1834-7819.1990.tb05875.x 